\title{
The Relief Effects of Ramelteon on Refractory Chronic Migraine: ACase Report
}

\author{
Yi-Cheng Hou ${ }^{1}$, Chien-Han Lai ${ }^{2,3}$ \\ ${ }^{1}$ Department of Nutrition, Taipei Tzu Chi Hospital, Buddhist Tzu Chi Medical Foundation, New Taipei City, ${ }^{2}$ Department of Psychiatry, Cheng \\ Hsin General Hospital, Taipei, ${ }^{3}$ Department of Biomedical Imaging and Radiological Sciences, National Yang-Ming University, Taipei, \\ Taiwan, ROC
}

\begin{abstract}
The selective melatonin receptor agonism effect of ramelteon is useful for insomnia. Here we wanted to present a refractory chronic migraine case, who had significant improvements in migraine after using ramelteon. The possible mechanism for the ramelteon in the migraine relief might be related to melatonin effects.
\end{abstract}

KEY WORDS: Ramelteon; Migraine disorders; Sleep wake disorders.

\section{INTRODUCTION}

The selective melatonin receptor agonism effect of ramelteon is useful for insomnia. ${ }^{1)}$ Here we wanted to present a refractory chronic migraine case, who had significant improvements in migraine after using ramelteon.

\section{CASE}

A 71 years old female patient suffered from chronic migraine for 2 decades.

She never had insomnia problems before the first onset of migraine. The migraine occurred 3-4 times per week and each episode persisted around 4-8 hours. The pulsating pain was localized at right half of the head. The precipitating factors were the stress, increase in physical activities and sudden cold weather. The relieving factors included warm temperature, slower pacing of life and comfortable environment. The moderate or severe pain intensity was aggravated by or causing avoidance of increased physical activity. The residual symptom between the migraine episodes were the head tense feeling, but not achieveing headache severity. The function remained well except avoiding exercise between migraine episodes. No family history of headache was mentioned. The diagnosis

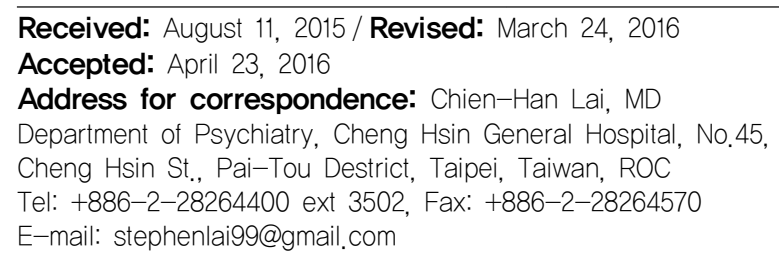

was made by the International Headache Society criteria for migraine. The computed tomography revealed no significant findings of other causes for headache, such as tumor or hemorrhage. She ever received many kinds of medications, such as aspirin, nonsteroidal anti-inflammatory drugs, triptans, ergots, anticonvulsants and glucocorticoids. However, the severity of migraine still remained moderate (Migraine Disability Assessment Test [MIDAS] scores, 19) and the associated symptoms were moderate headache, dizziness, nausea, and sensitivity to sound. The migraine also exacerbated her insomnia problems, with fragmented sleep and inadequate sleep duration as 1-2 hours (Insomnia Severity Index [ISI] score, 23). She didn't have any comorbid mental disorder except insomnia. Due to the fear of abuse potential related to hypnotics, she chose to receive ramelteon to relieve insomnia. The concurrent analgesic medicine for migraine was ibuprofen $400 \mathrm{mg} /$ day. After 2 weeks treatment of ramelteon 8 $\mathrm{mg}$ /day, her sleep duration prolonged to 4-5 hours with less fragmentation. In addition, her migraine severity started to decline (MIDAS scores, 11). After 6 months of ramelteon $8 \mathrm{mg}$ /day treatment, the migraine severity continued to relieve (MIDAS scores, 6) with stable sleeping quality (ISI score, 14).

\section{DISCUSSION}

In this case, we found significant improvements in migraine severity and insomnia after the use of ramelteon. Since the patient didn't have insomnia before the first onset of migraine. Therefore we could speculate that the ra-

(c) This is an Open-Access article distributed under the terms of the Creative Commons Attribution Non-Commercial License (http://creativecommons.org/licenses/by-nc/4.0) which permits unrestricted non-commercial use, distribution, and reproduction in any medium, provided the original work is properly cited. 
melteon had significant effects in migraine in such mild prolongation of sleep duration. The melatonin might relieve the headache via the following possible mechanisms, such as anti-inflammatory effect, free radical scavenging, reduction of pro-inflammatory cytokine, membrane stabilization, nitric oxide synthase activity and dopamine release inhibition, GABA and opioid analgesia potentitation, glutamate neurotoxicity protection, neurovascular regulation, ${ }^{2)}$ cytoprotection and antiallodynic action. ${ }^{3)}$ The pineal gland, the primary source of serotonin and melatonin, might also play a significant role in the analgesic effect. $\left.{ }^{4}\right)$ However, the clinical trial of melatonin administration showed no significant improvements in migraine severity, ${ }^{5}$ which suggested the modulation of melatonin receptor might be a better option for the analgesic effects. The modulation of serotonin system by the ramelteon would also relieve the pain sensation. ${ }^{2)}$ In addition, the chemical structure of ramelteon was similar with that of indomethacin, which is a kind of non-steroidal anti-inflammatory medicine. ${ }^{2)}$ In the study of animal model, the antinociceptive and antiallodynic actions of melatonin have been observed in different kinds of pathways, such as intrathecally or intracerebroventricular routes. The use of ramelteon, a kind of MT1 and MT2 agonist, could also re- lieve the pain even lack of free radical scavenger effects. ${ }^{3)}$ The state-of-art treatment of refractory chronic migraine also includes the ramelteon due to selective M1 receptor agonism. ${ }^{6}$ From the successful experience in this case, we can consider the use of ramelteon for refractory chronic migraine with insomnia.

\section{REFERENCES}

1. Reynoldson JN, Elliott E Sr, Nelson LA. Ramelteon: a novel approach in the treatment of insomnia. Ann Pharmacother 2008;42:1262-1271.

2. Peres MF, Masruha MR, Zukerman E, Moreira-Filho CA, Cavalheiro EA. Potential therapeutic use of melatonin in migraine and other headache disorders. Expert Opin Investig Drugs 2006; 15:367-375.

3. Srinivasan V, Pandi-Perumal SR, Spence DW, Moscovitch A, Trakht I, Brown GM, et al. Potential use of melatonergic drugs in analgesia: mechanisms of action. Brain Res Bull 2010;81:362-371.

4. Toglia JU. Melatonin: a significant contributor to the pathogenesis of migraine. Med Hypotheses 2001;57:432-434.

5. Alstadhaug KB, Odeh F, Salvesen R, Bekkelund SI. Prophylaxis of migraine with melatonin: a randomized controlled trial. Neurology 2010;75:1527-1532.

6. Lionetto L, Negro A, Palmisani S, Gentile G, Del Fiore MR, Mercieri $\mathrm{M}$, et al. Emerging treatment for chronic migraine and refractory chronic migraine. Expert Opin Emerg Drugs 2012;17:393-406. 